\title{
Análisis de los proyectos de investigación de financiación pública en Psicología del Deporte
}

\author{
Aurelio Olmedilla*, Enrique Ortega, Juan González y Diego Hernán-Villarejo
}

Universidad de Murcia

\begin{abstract}
Resumen: El objetivo del presente estudio fue analizar los Proyectos de Investigación $\mathrm{I}+\mathrm{D}+\mathrm{i}$ financiados por el Plan Nacional en el área de gestión de Psicología y en el área de gestión de Deporte, así como los concedidos por el Consejo Superior de Deportes. Para ello se registró de la página web del Ministerio de Innovación y Ciencia los proyectos vinculados con las ciencias del deporte $(\mathrm{n}=155)$, así como los vinculados con la psicología $(n=646)$. Además se registraron los proyectos concedidos por el Consejo Superior de Deportes (CSD) $(n=203)$. De cada uno de los proyectos concedidos se han analizado las siguientes variables: año de concesión, género del investigador principal, nombre del investigador principal, comunidad autónoma, universidad u organismo, cuantía de la concesión, tipología de proyectos, modalidad de proyectos de Psicología del Deporte y tipo de muestra de los proyectos de Psicología del Deporte. Los resultados indican que es mucho mayor la concesión de proyectos de Psicología del Deporte en las convocatorias del Plan Nacional gestionadas dentro del área de gestión de deporte y del Consejo Superior de Deportes, que los que son gestionados dentro del área de gestión de Psicología, y además en todos ellos es mucho mayor el porcentaje de hombres que de mujeres como investigador principal. Las comunidades autónomas de Cataluña, Comunidad Valenciana, Andalucía y Extremadura se reparten más del 70\% de los proyectos concedidos en Psicología del Deporte. Entre la Universidad Autónoma de Barcelona, la Universidad de Extremadura y la Universidad de Valencia, se distribuyen más de un tercio del total de proyectos de Psicología del Deporte. La modalidad de Psicología del Deporte con mayor número de proyectos es la de Salud y Calidad de Vida, seguida de Motivación y Emoción y de Psicobiología del Deporte.

Palabras clave: Psicología del Deporte, Proyectos de Investigación, Finan-
\end{abstract} ciación Pública.

\section{Introducción}

La creación del Espacio Europeo de Educación Superior (EEES), con sus pros y sus contras, ha provocado un profundo cambio en la universidad española, acentuado por el actual estado de crisis económica, que ha puesto a la universidad en un trance de inciertas consecuencias. En este contexto, y a pesar de él, la Conferencia de Rectores de Universidades Españolas (CRUE) en su informe La Universidad española en cifras (CRUE, 2011), indica que todo el proceso de creación del EEES ha revalorizado las competencias profesionales y científicas de las sociedades actuales, marcadas por la capacidad de innovar y adaptarse a nuevos entornos con rapidez y eficacia. Para Osuna (2009) los conceptos de calidad, excelencia, competencia, responsabilidad pública, transparencia y eficiencia están continuamente presentes en la educación superior europea actual. En definitiva, la cultura de la calidad y la mayor transparencia del sistema universitario son claves para rendir cuentas a los ciudadanos, que son en última instancia los que sufragan las universidades (Buela-

* Dirección para correspondencia [Correspondence address]: Aurelio Olmedilla Zafra. Universidad de Murcia. Facultad de Psicología. Departamento de Personalidad, Evaluación y Tratamiento Psicológico. Campus Universitario de Espinardo. 30800 Murcia (España). E-mail: olmedilla@um.es
Title: Analysis of draft publicly funded research in Sport Psychology.

Abstract: The aim of this study was to analyze the Research Projects $\mathrm{I}+\mathrm{D}+\mathrm{i}$ financed by the National Plan in the area of psychology and management in the area of Sport Management, as well as granted by the Sports Council. Thiswas recorded on the website of the Ministry of Innovation and Science projects related to sports science $(n=155)$ and those associated with psychology $(n=646)$. In addition there were projects awarded by the National Sports Council (CSD) $(\mathrm{n}=203)$. From each of the approved projects have analyzed the following variables: year of the grant, gender researcher, principal investigator name, Region, University or agency, the grant amount,project type, project mode of Psychology sport and type of sample projects of Sport Psychology. The results indicate that the grant is much larger project of Sport Psychology in the "Plan Nacional" managed within the area of sport management and the Sports Council, that are managed within the management area of psychology, and well in all of them is much higher percentage of men than women as principal investigator. The regions of Cataluña, Valencia, Andalucía and Extremadura are spread over $70 \%$ of the approved projects in Sport Psychology. Among the Autonomous University of Barcelona, the University of Extremadura and the University ofValencia are distributed over a third of all projects of Sport Psychology. The Psychology of Sport mode with more projects is the Health and Quality of Life, followed Motivation and Emotion and Psychobiology of Sport.

Key words: Sport Psychology, Research Projects, Public Funding.
Casal, Bermúdez, Sierra, Quevedo-Blasco, Guillén-Riquelme y Castro, 2010).

El creciente interés por la calidad en la educación superior ha provocado un conjunto de estudios para evaluar la investigación y la docencia que se realiza en las universidades españolas. La evaluación de la investigación se ha realizado contando con indicadores bibliométricos fiables, sobre todo en términos de relevancia, cantidad e impacto de esa investigación (Betz, 2010). Aunque se han utilizado diferentes criterios de evaluación (Buela-Casal, Bermúdez, Sierra, Quevedo-Blasco, Castro y Guillén-Riquelme, 2011) los proyectos de investigación han sido y son uno de los pilares básicos en los indicadores de la calidad de la Universidad en general (Buela-Casal, Bermúdez, Sierra, Quevedo-Blasco, y Castro, 2010; CRUE, 2011; International Ranking Expert Group, 2006), y del personal docente e investigador en particular (Buela-Casal y Sierra, 2007). En concreto, en la multitud de criterios de clasificación (ranking), a nivel nacional e internacional, el número de proyectos de $\mathrm{I}+\mathrm{D}+\mathrm{i}$ recibidos por una universidad es uno de los aspectos más valorados (BuelaCasal et al., 2010; Buela-Casal, Bermúdez, Sierra, QuevedoBlasco, Guillen-Riquelme y Castro, 2010). Tal es así, que en nuestro país no son pocos los trabajos de investigación en los que se plantean programas específicos de actuación en universidades, para incrementar la consecución de estos pro- 
yectos, así como diferentes procesos de evaluación interna que permiten dar a conocer limitaciones y posibles mejoras (Ortega, 2010).

Por otro lado, y a partir de la creación de la Agencia Nacional de Evaluación de la Calidad y Acreditación (ANECA), especialmente del programa Evaluación del Profesorado para la Contratación (PEP), y el de Acreditación nacional para el acceso a los cuerpos docentes universitarios (ACADEMIA), la dirección y participación en proyectos de investigación se ha convertido en una "necesidad" para la carrera universitaria. En concreto en el estudio de Buela-Casal y Sierra (2007) en el que participaron 1294 profesores universitarios $(72 \%$ profesores titulares con al menos un tramo de investigación y el resto catedráticos de universidad con al menos dos tramos de investigación), la dirección y participación en proyectos $\mathrm{I}+\mathrm{D}+\mathrm{i}$, fue uno de los aspectos más valorados (concretamente el $3^{\circ}$ ) por los profesores para la consecución de la acreditación nacional y para la acreditación de catedrático. Aunque estos datos tienen un importante valor cuantitativo, es necesario añadirles un enfoque cualitativo, utilizando aquellas metodologías y técnicas que sirvan a ambos aspectos, lo que sería recomendable para una evaluación más completa de la actividad científica (Sánchez-Gil y SanzCasado, 2011).

El campo de estudio de la actividad física y del deporte ha experimentado un incremento muy significativo en las dos últimas décadas (Valenciano, Antolín, Moreno, Devís y Villamón, 2003), tanto a nivel general (Devís, Valenciano, Villamón y Pérez-Samaniego, 2010; Valenciano, 2010; Villamón, Job, Valenciano y Devís, 2012) como en algunas áreas específicas (Olmedilla, Abenza, Ortega y Boladeras, 2011); no solo se ha producido un incremento muy importante en el número de artículos de investigación que tratan sobre el tema (Arbinaga, Aragón y Tejedor, 2010; Morales y Gálvez, 2012; Peñaranda, Garcés de los Fayos, Qiñones, Jara, y López, 2001; Valenciano, Devís y Villamón, 2005; Valenciano, Devís y Villamón, 2008), sino también en tesis y congresos (Kirk, 2010; Olmedilla, Ortega, Garcés de los Fayos, Jara y Ortín, 2009). Sin embargo, uno de los indicadores más relevantes del estado de la actividad científica en un área determinada deviene del número de proyectos de $\mathrm{I}+\mathrm{D}+\mathrm{i}$ del Plan Nacional.

La profesora Ferro (2009) realiza una extraordinaria aproximación histórica de los proyectos $\mathrm{I}+\mathrm{D}+\mathrm{i}$ en las ciencias del Deporte. Al realizar una breve revisión histórica entorno a los diferentes planes nacionales de $\mathrm{I}+\mathrm{D}$ relacionados con la actividad física y el deporte, indica que como consecuencia del hito de la celebración de los Juegos Olímpicos en Barcelona en 1992, se instaura en España, por primera vez un Programa Nacional de Investigaciones sobre Ciencias del Deporte, dentro del II Plan Nacional I+D (1992-1995). Tras este importante programa nacional, y como consecuencia de multitud de aspectos pero principalmente porque "las líneas presentadas tenían limitada repercusión práctica y eran escasos los grupos de científicos y los proyectos de investigación de calidad presentados" (Ferro, 2009, p. 1), se deja de apos- tar por políticas de investigación en el entorno del deporte. No es de nuevo hasta el año 2004, en el que se decide apostar por una nueva política de investigación en el ámbito de las ciencias del deporte, en concreto a través de la Acción Estratégica sobre Deporte y Actividad Física (2005-2008) dentro del V Plan Nacional de I+D+i (2004-2007). Tal y como la propia Ferro (2009) indica,

dicha iniciativa tuvo como objetivo el fomento de las actividades de investigación y desarrollo dirigidas a crear conocimiento científico y lograr avances técnicos y tecnológicos que contribuyan a generar y mejorar los productos y servicios que permitan una mejora de la calidad de vida de las personas (p. 1).

Los buenos resultados en esta acción estratégica, dieron lugar a que en el VI Plan Nacional (2008-2011) el Instituto de Salud Carlos III recogiera, dentro de su Acción Estratégica de Salud, un Subprograma de Salud, Deporte y Actividad Física (2008-2011). De nuevo los buenos resultados obtenidos, en relación a la cantidad y calidad de los proyectos presentados, ha dado lugar a que de nuevo entremos en el VI Plan Nacional I+D+i 2008-2011 como lo estábamos en el II Plan. Concretamente en el actual Programa Nacional de Proyectos de Investigación Fundamental, en el marco del VI Plan Nacional de Investigación Científica, Desarrollo e Innovación Tecnológica 2008-2011, se contemplan las siguientes convocatorias: a) Subprograma de Proyectos de Investigación Fundamental no orientada; b) Subprograma de Proyectos de Investigación Fundamental orientada a la transmisión del conocimiento a la empresa; c) Subprograma de Acciones Complementarias a Proyectos de Investigación Fundamental no orientada; d) Subprograma de Investigación Fundamental orientada a los Recursos y Tecnologías Agrarias en coordinación con las Comunidades Autónomas; y e) Acciones Complementarias, Investigación Aplicada, Acciones Complementarias y Acciones Especiales.

Sin lugar a dudas, una de las convocatorias más importantes es el Subprograma de Investigación Fundamental no orientada que, en nuestro caso, sustituiría a las Acciones Estratégicas anteriores. Además, cabe destacar como hito determinante en nuestro avance como ámbito científico, la aparición de un nuevo apartado: Área Temática de Gestión de Ciencias del Deporte, lo que supone que todos aquellos investigadores que apuesten por esta área, serán evaluados por una comisión de expertos investigadores del ámbito de las Ciencias del Deporte, además de la que también realicen los expertos de la ANEP; y se tendrá que competir entre proyectos de la misma área de gestión, si bien los criterios de evaluación son los mismos para todas las áreas del Subprograma.

Teniendo en cuenta todo lo anterior, una de las formas en las que se puede contemplar para evaluar o diagnosticar el estado actual en un campo de conocimiento científico, es el análisis de los proyectos de investigación. Por ello, el objetivo del presente estudio es analizar los Proyectos de Investigación $\mathrm{I}+\mathrm{D}+\mathrm{i}$ financiados por el Plan Nacional en el área de gestión de Psicología y en el área de gestión de Deporte, así 
como los concedidos por el Consejo Superior de Deportes, centrándonos básicamente en los Proyectos de Psicología del Deporte. Los aspectos a analizar han sido: a) Año de concesión; b) Sexo del investigador principal (IP); c) Nombre del IP; d) Comunidad autónoma; e) Universidad u organismo; f) Cuantía de la concesión; g) Tipología de proyectos; h) Modalidad de Psicología del Deporte; e i) Tipo de muestra.

\section{Método}

Se han recogido los proyectos de investigación vinculados con las ciencias de la actividad física y del deporte y con psicología $(n=1004)$. Para ello, de la página web del Ministerio de Innovación y Ciencia, dentro del Plan Nacional se recogieron todos los proyectos de investigación del área de gestión de Deporte (inician sus siglas con DEP), del Subprograma de Proyectos de Investigación Fundamental no orientada desde el año 2008 (año en el que se inicia el área de gestión de Deporte) al año $2012(n=77)$. De igual modo se registraron todos los proyectos pertenecientes a la convocatoria del Plan Nacional de las Acciones estratégicas de "Actividad física y deporte" del año 2005 y $2006(n=78)$. Por otro lado se ha recogido dentro del Plan Nacional todos los proyectos de investigación del área de gestión de Psicología (ini- cian sus siglas con PSIC), desde el año 2005 al año 2011 $(n=646)$. Finalmente del Consejo Superior de Deportes (CSD) se registraron el total de proyectos en sus diferentes modalidades, desde el año 2005 hasta el año 2012 ( $n=203)$.

De cada uno de los proyectos concedidos se han analizado las siguientes variables: a) Año de concesión; b) Sexo del IP; c) Nombre del IP; d) Comunidad autónoma; e) Universidad u organismo; f) Cuantía de la concesión; g) Tipología de proyectos (proyectos de deporte, proyectos de psicología; proyectos de psicología del deporte); h) Modalidad de Psicología del Deporte (motivación y emoción, entrenamiento psicológico, salud y calidad de vida, aprendizaje, control y desarrollo motor, modelos metodológicos y evaluación, iniciación deportiva, psicobiología del deporte, y lesiones y retirada deportiva); e i) Tipo de muestra (iniciación deportiva, alto rendimiento, entrenadores, adultos sedentarios, deportistas adultos, y tercera edad).

\section{Resultados}

En la Tabla 1, se recoge el número y porcentaje de proyectos de investigación de Psicología, de Deporte y de Psicología del Deporte, según convocatoria (Plan Nacional o CSD), y según el género del IP.

Tabla 1. Proyectos de Psicología, Deporte y Psicología del Deporte, según convocatoria y según género del IP.

\begin{tabular}{|c|c|c|c|c|c|}
\hline Variable & Categoría & $\begin{array}{c}\text { Proyectos } \\
\text { de Psicología }\end{array}$ & $\begin{array}{l}\text { Proyectos } \\
\text { de Deporte }\end{array}$ & $\begin{array}{c}\text { Proyectos } \\
\text { de Psicología del Deporte }\end{array}$ & Total Proyectos \\
\hline \multirow[t]{2}{*}{ Convocatoria } & Plan Nacional DEP y CSD & - & $\begin{array}{c}330 \\
(92.2 \%)\end{array}$ & $\begin{array}{c}28 \\
(7.8 \%)\end{array}$ & 358 \\
\hline & Plan Nacional PSIC & $\begin{array}{c}630 \\
(97.5 \%)\end{array}$ & - & $\begin{array}{c}16 \\
(2.5 \%)\end{array}$ & 646 \\
\hline \multirow[t]{2}{*}{ Género IP } & Masculino & $\begin{array}{c}219 \\
(64.0 \%)\end{array}$ & $\begin{array}{c}220 \\
(77.7 \%)\end{array}$ & $\begin{array}{c}30 \\
(71.4 \%)\end{array}$ & 462 \\
\hline & Femenino & $\begin{array}{c}123 \\
(36.0 \%)\end{array}$ & $\begin{array}{c}63 \\
(22.3 \%)\end{array}$ & $\begin{array}{c}12 \\
(28.6 \%)\end{array}$ & 195 \\
\hline
\end{tabular}

Los datos de la Tabla 1 señalan que tanto en número como en porcentaje, es mucho mayor la concesión de proyectos de Psicología del Deporte en las convocatorias del Plan Nacional gestionadas dentro del área de gestión de deporte (DEP) y del Consejo Superior de Deportes, que los que son gestionados dentro del área de gestión de psicología (PSIC). Por otro lado, también se aprecia que tanto en los Proyectos de Psicología, como de Deporte o de Psicología del Deporte, es mucho mayor el porcentaje de IP masculino que femenino. El porcentaje de IP masculinos es aun mayor en proyectos de Deporte, y algo menor en los proyectos de Psicología del Deporte y Psicología.

En la Tabla 2, se indica el nombre de los IP que han obtenido dos o más proyectos de investigación del Plan Nacional en el programa de gestión de Psicología.

Tabla 2. IP con dos o más proyectos del Plan Nacional en el programa de gestión de Psicología.

\begin{tabular}{lll}
\hline Alonso, Gumersinda & Fuentes, Luís J. & Muñiz, José \\
Carrera, Pilar & Gallo, Milagros & Ortet, Generos \\
Carretie, Luis & García, Gloria & Pellon, Ricardo \\
Cimadevilla, José M. & Lemos, Serafín & Pérez, Miguel \\
Colmenares, Fernando & Luciano, Carmen & Rodríguez, Antonio \\
Costa, Albert & Lupiañez, Juan & Vallejo, Guillermo \\
Echeburua, Enrique & Marti, Margarita & Vea, Joaquín José \\
Elosua, Paula & Miñarro, José & Vigil, Andrés \\
Fernández, Ángel & Munduate, Lourdes & \\
\hline
\end{tabular}


En la Tabla 3, se pueden ver los IP que han obtenido dos o más proyectos de investigación del Plan Nacional en el programa de gestión de Deporte y/o en proyectos del CSD.

En la Figura 1 se observa la distribución de los proyectos de investigación objeto de estudio concedidos a cada Comunidad Autónoma según el tipo de proyecto: de Psicología, de Deporte, o de Psicología del Deporte. En concreto, de la Figura 1 destaca que entre las comunidades autónomas de
Madrid, Cataluña, Andalucía y Comunidad Valenciana, se reparten más del 70\% de los proyectos concedidos en Psicología, y más del 50\% en Deporte. Por otro lado en Psicología del Deporte, además de Cataluña, Comunidad Valenciana y Andalucía, destaca Extremadura, con un total entre estas cuatro comunidades autónomas de más del $70 \%$ de los proyectos concedidos.

Tabla 3. IP con dos o más proyectos del Plan Nacional en el programa de gestión de Deporte y/o en proyectos del CSD.

\begin{tabular}{lll}
\hline Amejides, Elena & Gil, Susana & Puig, Nuria \\
Barrero, José & Grande, Ignacio & Rivero, Antonio \\
Bernal, Francisco J. & Izquierdo, Mikel & Salvadorgue, Fernando \\
Calderón, Francisco J. & Lucía, Alejandro & Sampedro, Javier \\
Casajús, José A. & Marón, Alfonso & Sillero, Manuel \\
Cecchini, José A. & Martín, Ángel & Suárez, Concepción \\
Cordente, Carlos A. & Martín, M Milar & Úbeda, Natalia \\
Cruz, Jaume & Mora, Ricardo & Valle Soto, Miguel E. \\
Del Corral, Francisco & Myro, Rafael & Vilanova, Ana \\
Del Vales, Javier & Orrego, Jorge & Villar, Fernando \\
Gallardo, Leonor & Ortega, Enrique & \\
García, Juan & Palao, José M. & \\
García, Tomás & Pérez, José A. & \\
\hline
\end{tabular}
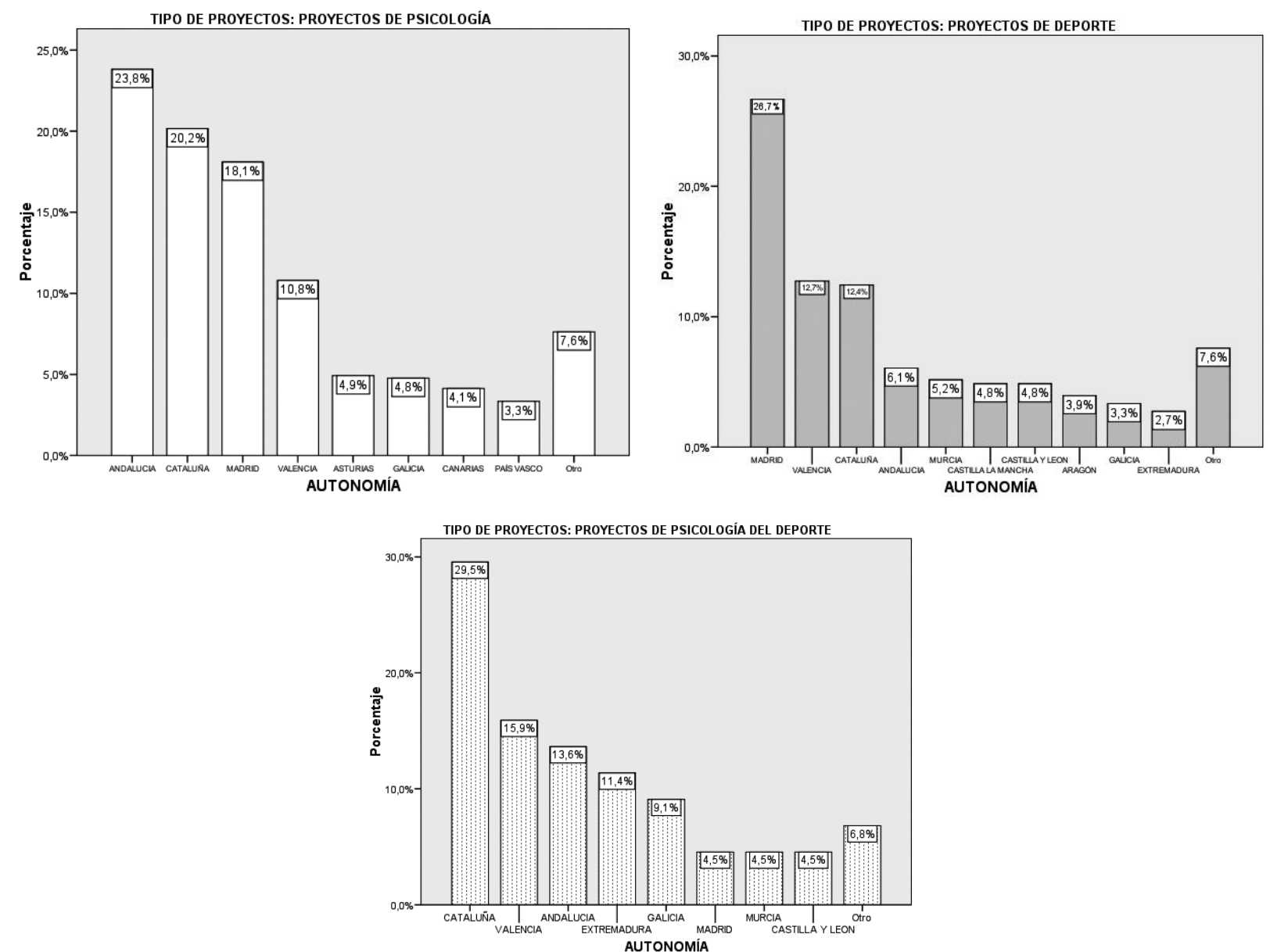

Figura 1. Porcentaje de proyectos concedidos a cada Comunidad Autónoma según el tipo de proyecto: de Psicología, de Deporte, o de Psicología del Depor- 
En la Figura 2 se observa la distribución de los proyectos de investigación objeto de estudio concedidos a cada Universidad u Organismo según el tipo de proyecto: de Psicología, de Deporte, o de Psicología del Deporte. En concreto, de la Figura 2 cabe destacar que en los proyectos de Psicología entre la Universidad Autónoma de Madrid (UAM), la Universidad de Granada (UGR), y la Universidad de Barcelona (UB), se reparten más de un tercio de los proyectos concedidos.

Dentro de los proyectos de Deporte, en la Figura 2 se aprecia que entre la Universidad Politécnica de Madrid
(UPM), la Universidad de Castilla La Mancha (UCLM), y la Universidad Europea de Madrid (UEM), se reparten el 19\% del total de los proyectos de Deporte. Finalmente, en la Figura 2 también se observa que entre la Universidad Autónoma de Barcelona, la Universidad de Extremadura y la Universidad de Valencia, se distribuyen más de un tercio del total de proyectos de Psicología del Deporte, destacando que existen un total de 17 organismos y/o universidades que al menos han recibido un proyecto de Psicología del Deporte.
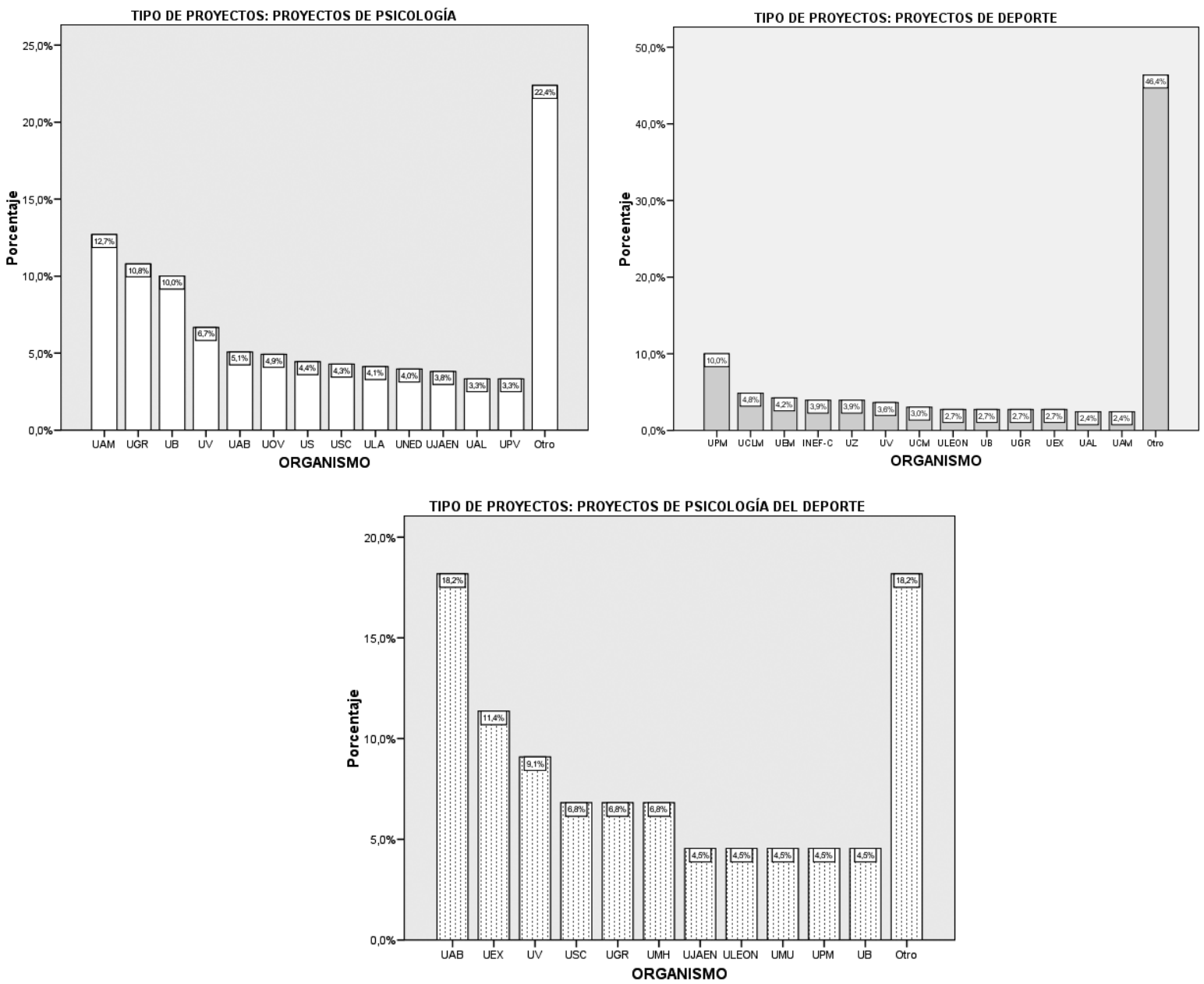

Figura 2. Porcentaje de proyectos concedidos a cada Universidad u Organismo según el tipo de proyecto: de Psicología, de Deporte, o de Psicología del Deporte.

En la Figura 3 se observa el valor medio concedido en euros según el tipo de proyecto: de Psicología, de Deporte, o de Psicología del Deporte. Además se aprecian las diferencias según género. En concreto de la Figura 3 se destaca que tanto a nivel general, como en proyectos donde el IP es un hombre, los proyectos que reciben una mayor subvención son los proyectos de Psicología, seguido de Psicología del Deporte, y finalmente los de Deporte. Cuando el IP es una mujer, los proyectos que más subvención reciben son los de Psicología del Deporte.

Por otro lado, de la Figura 3 destacan que tanto los Proyectos de Psicología, como los de Psicología del Deporte, los valores medios concedidos son superiores cuando el proyecto es dirigido por una mujer que cuando es dirigido por un hombre. Por el contrario, en los proyectos de Deporte, los valores medios subvencionados por proyecto son mayores cuando el IP es un hombre. 

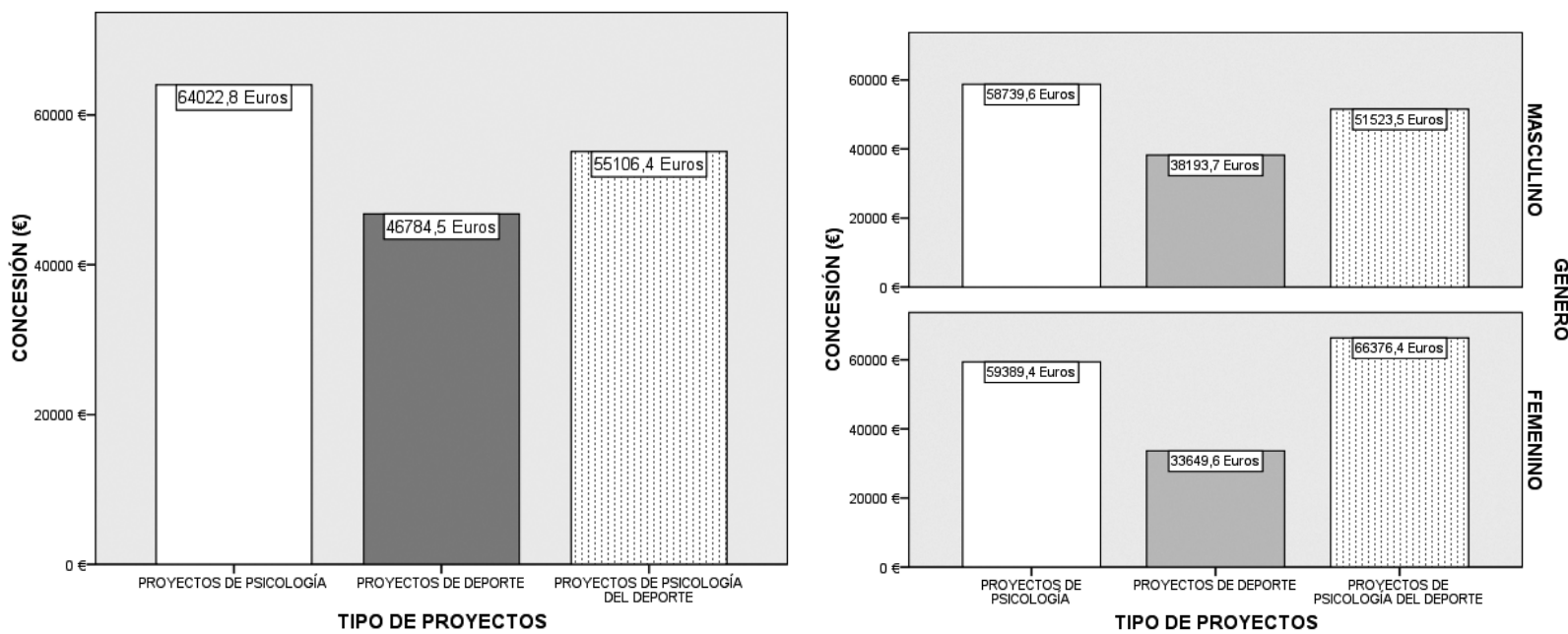

Figura 3. Valor medio concedido en euros según el tipo de proyecto: de Psicología, de Deporte, o de Psicología del deporte. Diferencias según género del IP.

En la Figura 4 se observan las subvenciones concedidas en euros según el tipo de proyecto: de Psicología, de Deporte, o de Psicología del Deporte. En concreto, se aprecia que en los proyectos de Psicología, la gran mayoría de ellos reciben subvenciones entre 20000 y 50000 euros, mientras que

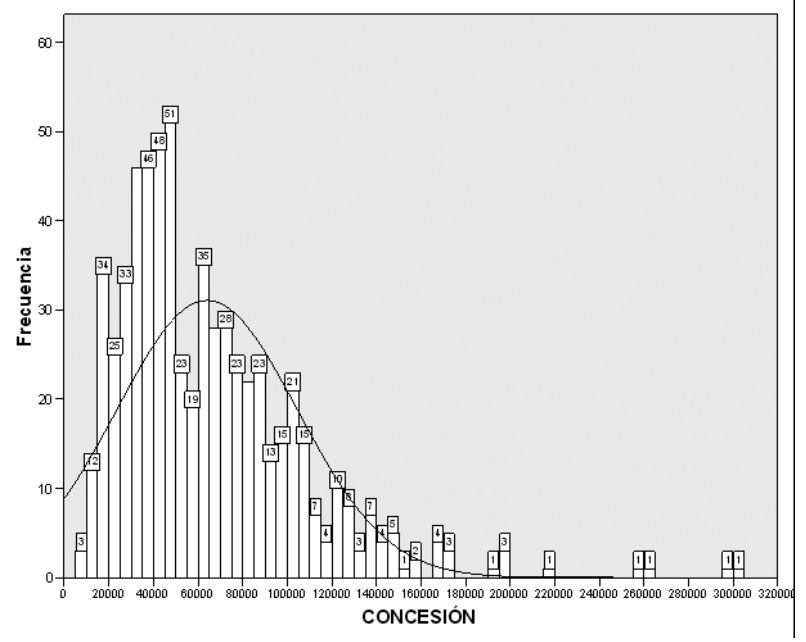

los proyectos de Deporte se sitúan entre 5000 y 20000 euros. En Psicología del Deporte se aprecia una gran heterogeneidad, existiendo dos grandes grupos: el primero de ellos subvencionados entre 5000 y 20000 euros, y el segundo con subvenciones entre 75000 y 85000 euros.

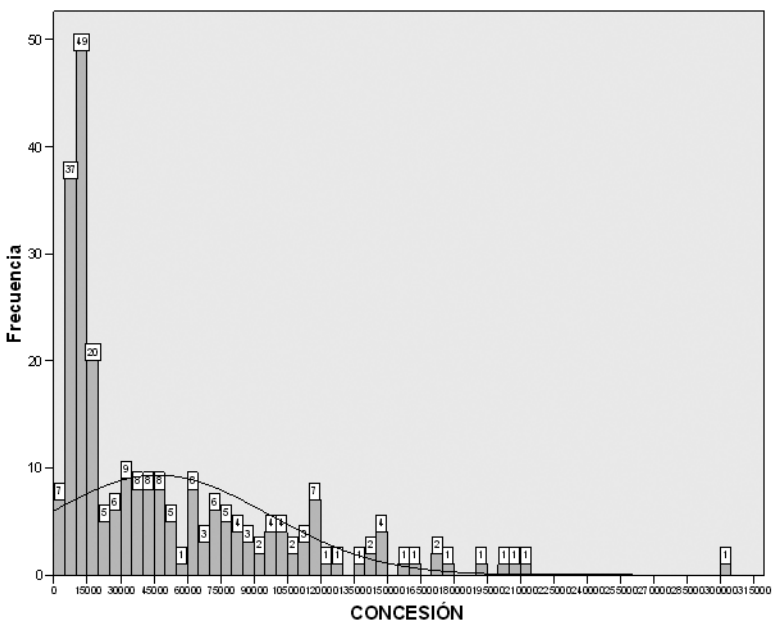

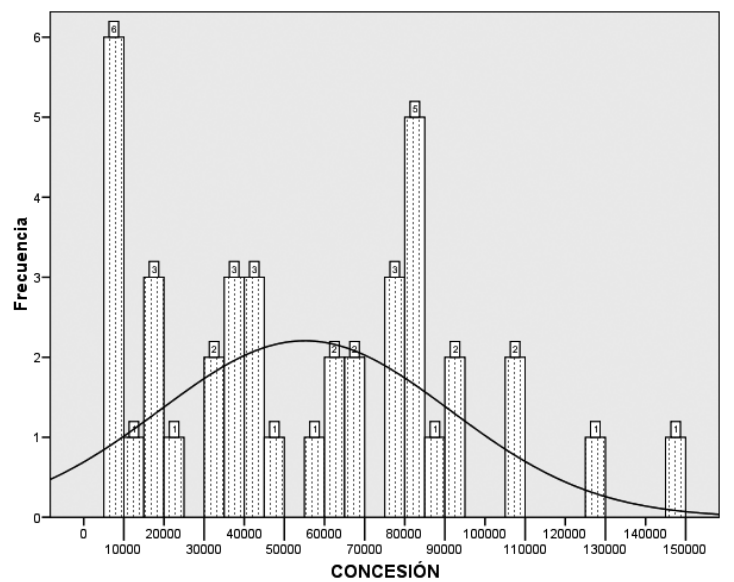

Figura 4. Frecuencias de subvenciones concedidas en euros según el tipo de proyecto: de Psicología, de Deporte, o de Psicología del Deporte. 
Finalmente, al analizar de manera más detallada los proyectos de Psicología del Deporte, en la Tabla 4 se indica el nombre de todos los IP. En concreto se aprecia que por lo menos 34 investigadores han recibido al menos un proyecto de investigación en Psicología del Deporte, destacando el
Dr. Jaume Cruz, de la Universidad Autónoma de Barcelona, y el Dr. Constantino Arce, de la Universidad de Santiago de Compostela, como los dos investigadores con mayor número de proyectos, concretamente con tres.

Tabla 4. IP con al menos un proyecto del Plan Nacional o del CSD, de la temática de Psicología del Deporte.

\begin{tabular}{lll} 
Alonso, José Ignacio & García, Tomás & Moreno, Juan Antonio \\
Andrés, Pilar & Godoy, Débora & Oña, Antonio José \\
Anguera, Ma Teresa & González, Javier & Pastor, Má Angeles \\
Arce, Constantino & Gusi, Narci & Portell, Isabel \\
Balaguer, Isabel & Guzmán, Lujan & Reyes del Paso, Gustavo A. \\
Baños, Rosa María & Iglesias, Damián & Rubio, Víctor \\
Capdevila, Lluis & Jiménez, Ana Concepción & Sáenz-López, Pedro \\
Córdova, Alfredo & Bernal, Jordi & Sicilia, Álvaro \\
Cruz, Jaume & De la Cruz, Juan Carlos & Suay, Ferran \\
Díaz, Arturo & Lavega, Pere & Torregrosa, Miquel \\
Escorihuela, Rosa María & Márquez, Sara & \\
Fernández del Olmo, Miguel & Moreno, Perla & \\
\hline
\end{tabular}

En esta misma línea, al observar la Figura 5, se puede apreciar que la modalidad de Psicología del Deporte con mayor número de proyectos es la de Salud y Calidad de Vida, seguida de Motivación y Emoción y de Psicobiología del Deporte, de manera que entre todos ellos suponen casi dos tercios del total de proyectos. Por contra destaca el bajo porcentaje de proyectos de Psicología del Deporte con Poblaciones Especiales, o de Lesiones, Iniciación Deportiva, y Aprendizaje, Desarrollo y Control Motor.

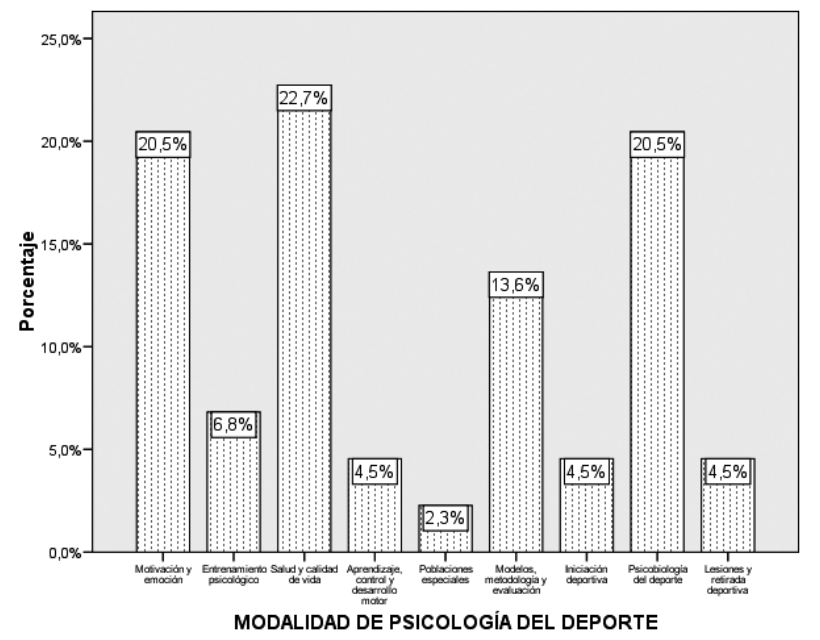

Figura 5. Porcentaje de proyecto de Psicología del Deporte, según modalidad.

Por otro lado al analizar dentro de los proyectos de Psicología del Deporte, el tipo de muestra objeto de estudio, en la Figura 6 se observa que en más de la mitad de los proyectos la muestra objeto de estudio o son deportistas adultos, o son adultos que no practican ningún tipo deporte, y en menor medida deportistas de alto rendimiento o de iniciación deportiva.

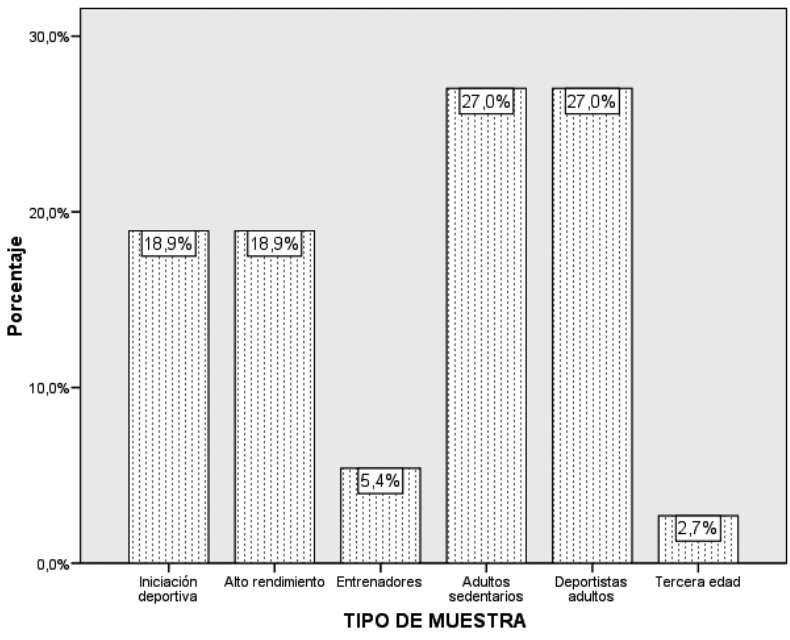

Figura 6. Porcentaje de proyecto de Psicología del Deporte, según tipo de muestra.

Por último, en la Figura 7 se puede observar el número de proyectos de Psicología del Deporte financiados, desde el año 2005 hasta el 2012. Como se puede apreciar el número de proyectos por año se mantiene entre 3 y 8 , si exceptuamos el año 2012, todavía sin concluir en el momento de elaboración del presente trabajo, y el año 2010 con un repunte hasta 12 proyectos. 


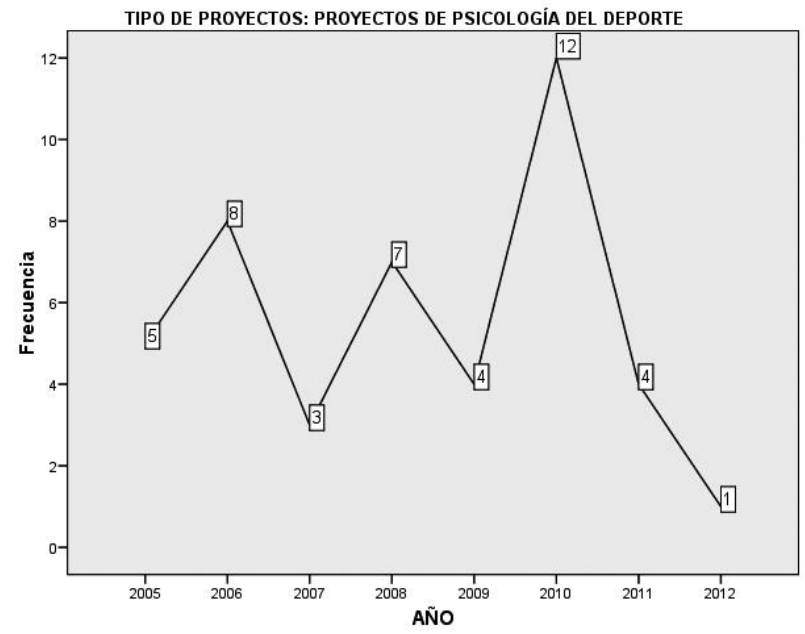

Figura 7. Evolución de proyectos de investigación de Psicología del Depor-

\section{Discusión}

En este trabajo se ha planteado analizar los Proyectos de Investigación $\mathrm{I}+\mathrm{D}+\mathrm{i}$ financiados por el Plan Nacional en el área de gestión de Psicología y en el área de gestión de Deporte, y los proyectos concedidos por el Consejo Superior de Deportes. Concretamente hemos centrado el análisis en los proyectos de Psicología del Deporte. Los resultados indican que en el intervalo del año 2005 al 2011, los proyectos financiados han sido 1004 , de los cuales solo un $4.38 \%$ son de Psicología del Deporte, además de éstos solo 16 han sido financiados por el Plan Nacional en el área de gestión de Psicología, lo que supone un escaso $2.5 \%$ del total. En el área específica de deporte, uniendo los planes del Plan Nacional DEP y el CSD, los proyectos de Psicología del Deporte suponen un $7.8 \%$ de 358 , es decir algo más que en PSIC, pero en cualquier caso los datos no son excesivamente optimistas sobre el área. Si tenemos en cuenta como criterio de análisis de la producción científica los proyectos de investigación, podemos observar que la Psicología del Deporte no parece excesivamente boyante, si bien se ha de considerar que es una especialidad joven.

Por otro lado, y aunque no se ha podido identificar el género de los IP en todos los Proyectos, en aquellos que sí se ha identificado los IP son hombres en su mayoría, lo que parece indicar que el sesgo de género sigue presente también en el liderazgo de los proyectos de investigación. Así, en los Proyectos de Psicología la mujer es IP en un 36\% de ellos, en los de Psicología del Deporte lo es en un 28.6\%, mientras que en los Proyectos de Deporte lo es en un porcentaje bastante menor, un $22.3 \%$, lo que parece mostrar que tanto en el ámbito deportivo como en el investigador, todavía la mujer se encuentra alejada de una posición de igualdad, lo que debe hacer que se insista en el desarrollo de políticas en investigación que incluyan la perspectiva de género. Aunque realmente no es fácil diseñar este tipo de políticas, sí es nece- sario tener la voluntad de hacerlo, ya que la incorporación de la mujer a la investigación, así como a otras actividades de la Universidad como la docencia o la gestión de la ciencia supone un progreso social y enriquece el conocimiento, ya que todas y todos ganamos incorporando otros modos de hacer, otras miradas (De Pablo, 2003; Selva, Pallarés y González, 2013). En este sentido algunos cambios en los criterios podrían llevarse a cabo, como por ejemplo la inclusión de un mayor número de mujeres en las comisiones evaluadoras para la concesión de Proyectos de Investigación. Tal y como indica Tarrach (2003),

la presencia de mujeres en estas comisiones es importante, no para que cuenten el número de publicaciones, para eso no hacen falta ni mujeres ni hombres, sino para que aporten su, normalmente, mejor comprensión de las dificultades añadidas con las que, no siempre, pero frecuentemente, se encuentra una mujer investigadora en comparación con su colega masculino (p.27).

Respecto a los datos de la distribución de Proyectos por Comunidades Autónomas, tanto los Proyectos de Psicología como los de Deporte están dominados por Andalucía, Cataluña, Madrid y Valencia, aunque destaca el hecho de que Madrid con porcentajes altos en ambos, solo aparece en el $6^{\circ}$ lugar en los Proyectos de Psicología del Deporte, y sobre todo sorprende que esto sea así cuando en los Proyectos de Deporte Madrid obtiene casi un 30\% de los Proyectos subvencionados, lo que indica que el número de Proyectos de Psicología del Deporte que se solicita es muy bajo; de hecho de las 11 primeras Universidades con Proyectos de Psicología del Deporte, de la Comunidad de Madrid solo aparece la Universidad Politécnica de Madrid, y con porcentajes bajos. Sin embargo, hay que tener en cuenta que este estudio analiza el número de Proyectos de Investigación concedidos, y no otros criterios como la productividad y la eficiencia, aspectos que sí fueron establecidos por Buela-Casal y su equipo en diferentes trabajos, realizados sobre el total de campos de investigación. Así, en el estudio de Buela-Casal et al. (2010) realizado sobre datos del año 2009, Cataluña lidera el ranking de comunidades autónomas en productividad respecto a los proyectos $\mathrm{I}+\mathrm{D}$, seguida de Baleares, Castilla-La Mancha y Madrid mientras que en eficiencia las cuatro primeras posiciones las ocupan Baleares, Cataluña, Cantabria y Aragón. Lo que concuerda, en parte, con los datos obtenidos en nuestro trabajo, ya que Cataluña aparece la $2^{\mathrm{a}}$ en Proyectos de Psicología, la $3^{a}$ en Proyectos de Deporte, y la $1^{\text {a }}$ en Proyectos de Psicología del Deporte. En el estudio de Valenciano, Devís y Villamón (2009), en el que analizan específicamente la producción científica de las Ciencias de la Actividad Física y el Deporte, a través de la afiliación aparecida en los 1786 artículos de 16 revistas españolas del campo en el periodo 1999-2005, las comunidades autónomas más productivas son Andalucía (14.61\%), Cataluña (14.11\%) y Madrid (10.97\%).

Respecto a los datos de la distribución de proyectos por Universidades se puede apreciar una mayor diversificación, sobre todo en los proyectos de Psicología, donde lidera la 
Universidad Autónoma de Madrid, y en los proyectos de Deporte, donde lo hace la Universidad Politécnica de Madrid pero con gran igualdad; no ocurre lo mismo en los proyectos de Psicología del Deporte, donde la Universidad Autónoma de Barcelona obtiene el 18.2\% ocupando la $1^{\text {a }}$ posición, seguida con cierta diferencia por la Universidad de Extremadura y la Universidad de Valencia. Posición que se ve refrendada en los datos aportados por Buela-Casal et al. (2011) en un estudio de la producción y productividad en investigación de las universidades públicas españolas durante el año 2010, donde la Universidad Autónoma de Barcelona aparece en los primeros puestos, concretamente la $3^{\text {a }}$, solo por detrás de la Universidad Complutense de Madrid y la Universidad de Barcelona; e igualmente refrendada por el Ranking de Shangai (Shangai Jiao Tong University, 2011), en el que aparece entre las 500 mejores universidades del mundo, con una evolución ascendente desde el año 2003. Por otro lado, en el mencionado trabajo de Valenciano et al. (2009) las universidades más productivas en la muestra de revistas españolas analizadas son la Universidad de Granada y la Universidad de León.

Respecto a la cuantía de la subvención de los proyectos, los resultados han señalado que los Proyectos de Psicología son los mejor valorados, seguidos por los de Psicología del Deporte y por los de Deporte, en último lugar. Es de destacar que tanto los Proyectos de Psicología, como los de Psicología del Deporte, los valores medios concedidos son superiores cuando el proyecto es dirigido por una mujer que cuando es dirigido por un hombre. Por el contrario, en los proyectos de Deporte, los valores medios subvencionados por proyecto son mayores cuando el IP es un hombre. En cualquier caso, el análisis de las subvenciones debe girar en torno a los conceptos de productividad y eficiencia, buscando la excelencia y competitividad de la universidad, incrementando así la calidad de la formación y de la investigación que se realiza en las universidades españolas (Osuna, 2009).

\section{Referencias}

Arbinaga, F., Aragón, J.D. y Tejedor, R. (2010). Análisis bibliométrico de la Revista de Psicología del Deporte (1992-2009). Revista de Psicología del Deporte, 19(2), 231-245.

Betz, B. (2010). Análisis de las fortalezas institucionales para la contratación de investigadores. Aula Abierta, 38, 65-74.

Buela-Casal, G. y Sierra, J.C. (2007). Criterios, indicadores y estándares para la acreditación de profesores titulares y catedrático de Universidad. Psicothema, 19(4), 537-551.

Buela-Casal, G., Bermúdez M.P., Sierra, J.C., Quevedo-Blasco, A. y Castro, A. (2010). Ranking de 2009 en investigación de las universidades públicas españolas. Psicothema, 22(2), 171-179.

Buela-Casal, G., Bermúdez M.P., Sierra, J.C., Quevedo-Blasco, A., GuillénRiquelme, A. y Castro, A. (2010). Relación de la productividad y eficiencia en investigación con la financiación de las comunidades autónomas españolas. Psicothema, 22(4), 924-931.

Buela-Casal, G., Bermúdez, M.P., Sierra, J.C., Quevedo-Blasco, R., Castro, A. y Guillén-Riquelme, A. (2011). Ranking de 2010 en producción y productividad en investigación de las universidades públicas españolas. Psicothema, 23(4), 527-536.
Respecto a la modalidad de los Proyectos de Investigación de Psicología del Deporte, los investigadores han ejecutado Proyectos en el ámbito de la Salud y Calidad de Vida, seguida muy de cerca por Motivación y Emoción y por Psicobiología del Deporte. Comparando estos datos con los del trabajo de Olmedilla et al. (2009), en el que muestran la evolución de los congresos nacionales de Psicología del Deporte realizados en España desde 1999 hasta 2008, es de destacar que la modalidad Salud y Calidad de Vida pasa de un $4^{\circ}$ puesto en el ranking de esta evolución, al primer puesto en Proyectos de Psicología del Deporte, lo que parece indicar la importancia que ha adquirido esta temática en estos últimos años. Además la modalidad Motivación y Emoción ocupa el $2^{\circ}$ puesto en ambos rankings, y la Psicobiología del Deporte está mucho mejor situada en los Proyectos ( $3^{\circ}$ puesto) que en los congresos analizados ( $9^{\circ}$ puesto). Por otro lado, destaca el bajo porcentaje de proyectos de Psicología del Deporte con Poblaciones Especiales, de Lesiones, Iniciación Deportiva, y Aprendizaje, Desarrollo y Control Motor.

Por último, la evolución de los Proyectos de Psicología del Deporte, desde el año 2005 hasta el 2012, todavía no concluido, muestra cierta regularidad (entre 4 y 8 proyectos por año, si exceptuamos el último año 2012, donde aparece un solo proyecto, y el año 2010 que aparece con 12), con un total de 44 proyectos y una media de 6.14 proyectos por año. Regularidad que por un lado parece mostrar cierta tendencia a su consolidación, aunque se requiere un mayor protagonismo de los grupos de investigación en Psicología del Deporte, generalmente, salvo casos excepcionales (como el grupo de Jaume Cruz, o el de Isabel Balaguer), muy jóvenes, o de reciente constitución como el de Aurelio Olmedilla en la Universidad de Murcia, o el de Víctor Rubio en la Universidad Autónoma de Madrid, y que necesitarán de gran perseverancia, rigurosidad y de planteamientos coordinados entre dos o más grupos de investigación, para obtener subvenciones de sus proyectos, sobre todo en el actual estado de cosas.

Conferencia de Rectores de Universidades Españolas, CRUE (2011). La Universidad española en cifras, 2010. Recuperado el 17 de julio de 2012 de http://www.crue.org/Publicaciones/UEC.html.

De Pablo, F. (2003). Nosotras también investigamos. Quark: Ciencia, medicina, comunicación y cultura, 27.

Devís, J., Valenciano, J., Villamón, M. y Pérez-Samaniego, V. (2010). Disciplinas y temas de estudio en las ciencias de la actividad física y el deporte. Revista Internacional de Medicina y Ciencias de la Actividad Física y el Deporte 10(37), 150-166.

Ferro, A. (2009). Las Ciencias del Deporte y la Política Científica Española. Revista Internacional de Ciencias del Deporte, 5(14), 1-2.

International Ranking Expert Group (2006). Purpose, criteria, and procedure. Bruselas: IREG, Observatory on Academic Ranking and Excellence.

Kirk, D. (2010). O porquê de investigar: estado atual e tendências futuras nas pesquisas em Educação Física. Movimento, 16(2), 11-43.

Morales, V., y Gálvez, P. (2012). Nuevas tecnologías en gestión: informatización del CECASDEP (Cuestionario de la evaluación de la calidad percibida en servicios deportivos. Cuadernos de Psicología del Deporte, 12(1), 159-162 
Olmedilla, A., Ortega, E., Abenza, L. y Boladeras, A. (2011). Lesiones deportivas y psicología: una revisión (2000-2009). Cuadernos de Psicología del Deporte, 11(1), 45-57.

Olmedilla, A., Ortega, E., Garcés de los Fayos, E.J., Jara, P. y Ortín, F.J. (2009). Evolución de la investigación y de la aplicación en Psicología del Deporte, a través del análisis de los Congresos Nacionales de Psicología del Deporte (1999-2008). Cultura, Ciencia y Deporte, 4(10), 15-23.

Ortega, E. (2010). Proyecto Docente e Investigador. Universidad de Murcia.

Osuna, E. (2009). Calidad y financiación de la universidad. Revista de Investigación en Educación, 6, 133-141.

Peñaranda, M., Garcés de los Fayos, E.J., Qiñones, E., Jara, P. y López, J.J. (2001). Un acercamiento a la comprensión de la psicología del deporte en España: Análisis de la Revista de Psicología del Deporte 1992-1999. Revista de Psicología del Deporte, 10(1), 69-81.

Sánchez-Gil, S. y Sanz-Casado, E. (2011). Patrón de investigación de los IPs de propuestas de investigación del Plan Nacional de $\mathrm{I}+\mathrm{D}+\mathrm{i}$ en el área de Ciencias Sociales 2004-2007 y calificadas como excelentes por la ANEP. En L. Cabello y M.P. Bermúdez (Comps.), Evaluación de la Calidad de la Educación Superior y de la Investigación (VIII Foro). Granada: Asociación Española de Psicología Conductual (AEPC).

Selva, C., Pallarés, S. y González, M.D. (2013). Una mirada a la conciliación a través de las mujeres deportistas. Revista de Psicología del Deporte, 22(1), 69-76.

Shangai Jiao Tong University (2011). Academic Ranking of World Universities. Recuperado el 17 de julio de 2012 de http://www.arwu.org/.
Tarrach, R. (2003). Investigación científica desde la perspectiva de género. Quark: Ciencia, medicina, comunicación y cultura, 27.

Valenciano, J. (2010). Producción científica y temas de investigación en educación física y deportes. En $V$ Congreso Internacional XXVT Congreso $\mathrm{Na}$ cional Educación Física Docencia, innovación e investigación en educación física. Barcelona: INDE.

Valenciano, J., Antolín, L., Moreno, A., Devís, J. y Villamón, M. (2003). Las revistas científico-técnicas españolas de las Ciencias de la Actividad Física y el Deporte: Inventario y análisis de calidad de contenido y difusión. Revista Española de Documentación Científica, 26(2), 177-190.

Valenciano, J., Devís, J. y Villamón, M. (2005). Análisis de la visibilidad de las revistas científico-técnicas españolas de Ciencias de la Actividad Física y el Deporte. Revista de Psicología del Deporte, 14(2), 253-267.

Valenciano, J., Devís, J. y Villamón, M. (2008). Análisis comparativo de la calidad de las revistas científico-técnicas españolas de Ciencias de la Actividad Física y el Deporte. Information Research, 13(1), 337.

Valenciano, J., Devís, J. y Villamón, M. (2009). Autoria institucional de los trabajos publicados en revistas españolas de ciencias del deporte (19992005). Cultura, Ciencia y Deporte, 10(5), 7-14.

Villamón, M., Job, I., Valenciano, J. y Devís, J. (2012). Estudio comparativo de cinco revistas de Ciencias del Deporte indizadas en WoS. Revista de Psicología del Deporte, 21(2), 281-287.

(Articulo recibido: 18-7-2012; revisado: 22-10-2012; aceptado: 15-12-2012) 